\title{
Dynamic mechanical relaxation of lightly cross-linked epoxidized natural rubber
}

\author{
W.B. Liau* \\ Institute of Materials Science and Engineering, National Taiwan University, Taipei, Taiwan, ROC \\ Received 30 January 1998; revised 23 March 1998; accepted 7 April 1998
}

\begin{abstract}
Ahstract
The isochronal mechanical relaxation spectra of lightly cross-linked epoxidized natural rubbers (ENR) were measured at a frequency of $1 \mathrm{~Hz}$ and the results analyzed using the empirical Havriliak-Negami (HN) equation. The HN equation fitted the relaxation spectra very well. The fitting parameters, $\alpha$ and $\beta$, obtained from the data fitting were used to interpret the relationship between molecular motions and structure of polymers based on the model proposed by Schönhals and Schlosser. Near the glass transition, the molecular motions of ENR in both the high and low frequency regions were not influenced by the level of cross-linking due to the low cross-linking density. Comparing with the natural rubber (NR), the epoxide groups of ENR promoted steric interactions and enhanced the inter-molecular cooperative motion. The activation energy of molecular motions for ENR was larger than observed for NR. The values of $\alpha$ and $\alpha \beta$ of ENR were less than observed for NR. According to the model proposed by Schönhals and Schlosser, $\alpha \beta$ was correlated with the local intra-molecular motions and $\alpha$ was correlated with the inter-molecular motions. The results suggest that the epoxide groups broaden the intra- and inter-molecular relaxation dispersion. (C) 1998 Elsevier Science Ltd. All rights reserved.
\end{abstract}

Keywords: Molecular motion; Havriliak-Negami equation; Dynamic mechanical relaxation

\section{Introduction}

Epoxidized natural rubber (ENR) is a relatively new rubber material, though the epoxidation of natural rubber (NR) is not a new idea for transforming natural rubber into a new material [1]. The epoxidation of natural rubber can be performed by peracid, generated from the reaction of formic acid and hydrogen peroxide, on the rubber latex [2]. There are now two commercial forms of epoxidized natural rubber available, ENR-25 ( 25 mol\% epoxidized) and ENR-50 ( $50 \mathrm{~mol} \%$ epoxidized). The properties of such materials are gradually changed with increasing degree of epoxidation [3]. The air permeability decreases, whereas hysteresis, oil resistance and wet traction increase with degree of epoxidation. In addition, ENR can undergo strain crystallization [4] like natural rubber and hence has superior tensile and fatigue properties. Such a property change is due to the modification of the chemical structure of natural rubber. ENR can be regarded as a random copolymer of 2methyl-1,2-epoxy-1,4-butanediyl and 2-methyl-1-butenylene. Certainly, such backbone structure modification of a

\footnotetext{
*Tel.: (02)-2362-61 19; fax: (02)-2363-4562; c-mail: wbliau@ccms.ntu.c-
}

polymer has a significant influence on its relaxation behaviour. Except for the chemical structure modification, the vulcanizate structure of ENR is also different from natural rubber. In ENR, the cyclic sulfides is not formed. The process of NR vulcanization with sulfur-accelerator systems involves the cross-linking of the polymer chains by monoand/or polysulfide bridges. An interesting side reaction along with the vulcanization found in NR is that the heterocyclic groups, cyclic sulfides, are formed randomly in the main chain [5-9]. The heterocyclic groups in the main chain have a profound effect on the relaxation behaviour [10-13]. In ENR, the sites of vulcanization are the same as in natural rubber [14-16]: in the methyl and methylene carbons allylic to the double bond. However, the double bonds may be isolated, having epoxide groups on either side, or having one adjacent epoxide group, the latter situation being twice as frequent as the former in the case of ENR-50. It was suggested that such a structure inhibited the formation of the heterocyclic group, cyclic sulfides $[14,16]$. It is the purpose of this work to study the structure effect on the relaxation behaviour near the glass transition of ENR by comparing it with the relaxation behaviour of natural rubber. At the same time, the level of cross-linking effects on the relaxation behaviour near the glass transition of ENR 
will be examined. In this work, the dynamic mechanical relaxation behaviours will be studied based on the model proposed by Schönhals and Schlosser and the molecular motions will be correlated with the structure of the polymers.

\section{Background}

Dielectric and mechanical relaxation methods are common probes for the study of molecular motions and structure. In this study, the mechanical relaxation behaviours of ENR are measured and the discussion is concentrated on the relationship between molecular motions and structure near the glass transition temperature.

Empirical functions have commonly been used to analyze the shape of the dielectric relaxation spectrum. Two of the most frequently used empirical equations are the Havriliak Negami (HN) [17] and the Kohlrausch-WilliamsWatts(KWW) [18,19] equations. Both equations have been successfully applied to the study of the relationship between the structure and relaxation behaviours of natural rubbers $[10,20]$. In this work, the analysis strategy for the mechanical relaxation spectra follows that of a previous paper [10]. The HN equations for the complex dielectric constant, $\varepsilon^{*}$, can be written as

$\varepsilon^{*}=\varepsilon_{\mathrm{u}}+\frac{\varepsilon_{\mathrm{r}}-\varepsilon_{\mathrm{u}}}{\left[1+\left(\mathrm{i} \omega \tau_{0}\right)^{\alpha}\right]^{\beta}}$

where $\varepsilon_{\mathrm{u}}$ and $\varepsilon_{\mathrm{r}}$ are the unrelaxed and relaxed values of the dielectric constants, $\tau_{0}$ is the mean relaxation time and $\omega$ is the angular frequency. In Eq. (1), $\alpha$ and $\beta(0<\alpha \leq 1,0<$ $\alpha \beta \leq 1)$ are adjustable fitting parameters. The HN equation was developed from the equation of single relaxation time model that was proposed by Debye [21].

It is quite common in dielectric studies to fit the relaxation spectrum with the HN equation. Although this is not so common for mechanical studies, it has been shown that the $\mathrm{HN}$ equation fits the mechanical relaxation spectrum as well as the dielectric one, including isochronal scans $[10,22,23]$. For mechanical relaxation, the $\mathrm{HN}$ equation is modified as

$E^{*}=E_{\mathrm{u}}+\frac{E_{\mathrm{r}}-E_{\mathrm{u}}}{\left[1+\left(\mathrm{i} \omega \tau_{0}\right)^{\alpha}\right]^{\beta}}$

where $E_{\mathrm{u}}$ and $E_{\mathrm{r}}$ are the unrelaxed and relaxed moduli of the polymer, respectively. Again, $0<\alpha \leq 1$ and $0<\alpha \beta \leq 1$.

Usually, the parameters $\left(E_{\mathrm{u}}, E_{\mathrm{r}}, \tau_{0}, \alpha\right.$ and $\left.\beta\right)$ of the $\mathrm{HN}$ equation are determined from a single isothermal relaxation spectrum. However, the relaxation of the polymer is so broad in the frequency or time domain at most temperatures that even a very wide ranging (in frequency or time) isothermal measurement covers a small part of the relaxation. It is necessary to determine parameters from several isothermal relaxation spectra to obtain sufficient information. Another way to achieve the same results is to regard the parameters $E_{\mathrm{u}}, E_{\mathrm{r}}, \alpha$ and $\beta$ as linear functions of temperature:

$$
\begin{aligned}
& E_{\mathrm{u}}=E_{\mathrm{u}}^{o}+E_{\mathrm{v}}^{s}\left(T-T_{\mathrm{u}}\right) \\
& E_{\mathrm{r}}=E_{\mathrm{r}}^{o}+E_{\mathrm{r}}^{s}\left(T-T_{\mathrm{r}}\right) \\
& \alpha=\alpha^{o}+\alpha^{s}\left(T-T_{\alpha}\right) \\
& \beta=\beta^{o}+\beta^{s}\left(T-T_{\beta}\right)
\end{aligned}
$$

where $T_{\mathrm{u}}, T_{\mathrm{r}}, T_{\alpha}$ and $T_{\beta}$ are arbitrarily chosen reference temperatures, the superscript $o$ indicates the value at an arbitrarily chosen reference temperature $T_{p}$, and the superscript $s$ indicates a linear temperature coefficient. The relaxation time, $\tau_{0}$, follows either an Arrhenius relation

$\log \tau_{0}=A / T+B$

or the WLF relation

$\log \tau_{0}=A /\left(T-T_{\infty}\right)+B$

Although the HN equation has been successfully used to fit the dielectric and mechanical relaxation data, it is still limited in correlating the parameters of the $\mathrm{HN}$ equation with the molecular motions and structure of polymer chains. A model proposed by Schönhals and Schlosser [24-26] has been successfully applied to explain the relationship between the structure and molecular motion near the glass transition temperature for natural rubber [10]. Schönhals and Schlosser suggested that the molecular motions of polymer in the glass transition are controlled by both intra- and inter-molecular interactions. When one considers the scale of molecular motion, the model suggests that in the high frequency region, i.e. $\omega \tau \gg 1$, the relaxation is controlled by the local chain dynamics and in the low frequency region, i.e. $\omega \tau \ll 1$, the relaxation is controlled by the intermolecular interaction. From Eq. (1) or Eq. (2), the slope of relaxation loss peak at the high frequency side in the plot of $\log (\operatorname{loss}$ peak) versus $\log (\omega \tau)$ is proportional to the product $-\alpha \beta$, and at the low frequency, the slope is proportional to $\alpha$. Therefore, the parameter $\alpha$ can be correlated to the inter-molecular dynamics and the product $\alpha \beta$ can be correlated to very local intra-molecular dynamics of the polymer [27].

\section{Experimental section}

The ENR (50 mol\% epoxidized, ENR-50), 2-mercapto benzothiazoles, 2-benzothiazole disulfide, $\mathrm{ZnO}$, stearic acid and sulfur were mixed on a two roll mill. Three samples with different cross-linking density (ENR-S04, ENR-S08 and ENR-S10) were prepared under the same conditions. The only difference between these samples is the amount of sulfur in the sample: based on ENR, the amount of sulfur in each sample is 0.4, 0.8 and $1 \mathrm{phr}$ for ENR-S04, ENR-S08 and ENR-S10, respectively. The recipes for the samples are listed in Table 1. Vulcanization was carried out by heating the samples up to $165^{\circ} \mathrm{C}$ for $10 \mathrm{~min}$. Then the sample was 
Table 1

The recipes for the samples

\begin{tabular}{lllllll}
\hline Sample & $\begin{array}{l}\text { Epoxidized natural } \\
\text { rubber }\end{array}$ & Sulfur & $\mathrm{DM}^{a}$ & $\mathrm{M}^{b}$ & ZnO & Stearic acid \\
\hline ENR-S04 & 100 & 0.4 & 0.2 & 0.1 & 1.0 & 0.5 \\
ENR-S08 & 100 & 0.8 & 0.2 & 0.1 & 1.0 & 0.5 \\
ENR-S10 & 100 & 1.0 & 0.2 & 0.1 & 1.0 & 0.5 \\
\hline
\end{tabular}

${ }^{a} \mathrm{DM}$ : 2-benzothiazole disulfide.

${ }^{b} \mathrm{M}: 2$-mercapto benzothiazoles.

cut into strips (typically $30 \times 10 \times 3 \mathrm{~mm}$ ) for the measurement of mechanical relaxation behaviour. The dynamic mechanical relaxation measurement was carried out in tension using a DMA (TA instrument Co.) with fixed mode $(1 \mathrm{~Hz})$ and $5^{\circ} \mathrm{C} \mathrm{min}^{-1}$.

It was noted that conventional high-sulfur vulcanite of ENR had a poor aging character [14,16]. On aging, the sulfur-containing acids, by-products of the aging process, induced the ring-opening cross-linking reaction of the epoxide group with the formation of an ether cross-link. To avoid this, the sulfur content of ENR was suggested to be low [16]. The previously reported [10] relaxation spectrum of NR04, whose sulfur content was $0.4 \mathrm{phr}$, was used for comparison in this study.

\section{Phenomenological data fitting}

In order to correlate the paramelers of the $\mathrm{HN}$ equation with the molecular motions and structure of the polymer chains, curve fitting of the relaxation spectrum was carried out using a non-linear least-squares optimization of the fit of the $\mathrm{HN}$ equation to both the experimental storage and the loss moduli isochronal curves. In this study, the discussion is concentrated on the glass relaxation. In the glass transition region, the drop in storage moduli is dramatic. Thus, the object function for minimization is written as

$$
\begin{aligned}
f= & \sum\left(\log E_{\text {cal }}^{\prime}(i)-\log E_{\text {exp }}^{\prime}(i)\right)^{2} \\
& +\sum\left(\log E_{\text {cal }}^{\prime \prime}(i)-\log E_{\text {exp }}^{\prime \prime}(i)\right)^{2}
\end{aligned}
$$

where $E^{\prime}$ and $E^{\prime \prime}$ are the storage and loss moduli, the subscripts 'cal' and 'exp' indicate the calculated value from Eq. (2) and the experimental value of the storage and loss moduli, respectively. The object function ignores the connection between $E^{\prime}$ and $E^{\prime \prime}$ occasioned by a KramersKronig-like relation. Minimization of the object function was carried out using Marquardt's method.

Since the fitting process is an iterative one, an initial guess for the parameters must be prepared. There are 10 parameters, $E_{\mathrm{u}}^{o}, E_{\mathrm{u}}^{s}, E_{\mathrm{r}}^{o}, E_{\mathrm{r}}^{s}, \alpha^{o}, \alpha^{s}, \beta^{o}, \beta^{s}, A, B$, for the glass transition to be determined from curve fitting of the relaxation spectrum. Since there are around 300 experimental data points for an isochronal relaxation spectrum, the determination of parameters usually proceeds satisfactorily. There is no constraint on the parameters during the curve fitting.

\section{Results and discussion}

Figs 1 and 2 show the isochronal storage and loss moduli of ENR samples along with the best fit of Eq. (2) (only the glass transition of the relaxation is shown). They show the typical relaxation spectrum of lightly cross-linked materials such as NR. The storage moduli is dropped from $\sim 10^{9}$ to $\sim 10^{6} \mathrm{~Pa}$ in the glass transition region. The glass transition temperatures are $-3.63,-0.66$ and $-0.21^{\circ} \mathrm{C}$ for ENRS04, ENR-S08 and ENR-S10, respectively. The glass transition temperature of sulfur-vulcanized ENR is about $50 \mathrm{~K}$ higher than the glass transition temperature of sulfur-vulcanized natural rubber reported in a previous paper [10]. This result is very consistent with the result reported by Brydson [28], who mentioned that the glass transition temperature of ENR was increased by approximately $1 \mathrm{~K}$ per mole\% epoxidized. Since the level of crosslinking is very low, the relative high glass transition temperature of ENR is due to the steric interference caused by the epoxide groups. The increase in glass transition temperature with increasing sulfur content for ENR is much less than that observed for NR. According to McCrum [7], a major factor in the increase of the glass transition temperature for sulfur-vulcanized NR is the formation of hetrocyclic

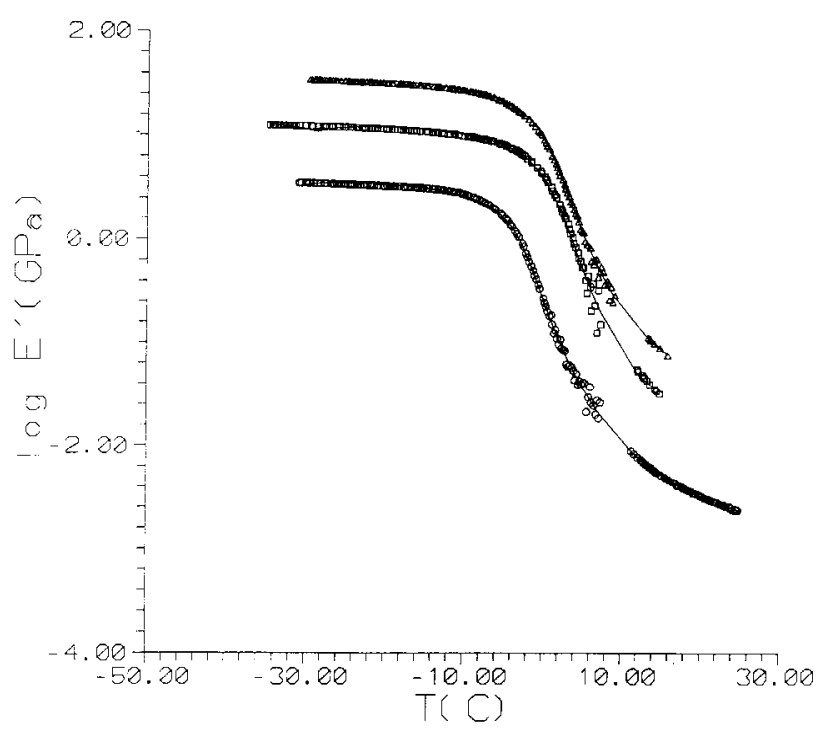

Fig. 1. Isochronal plot of the storage moduli measured for (a) ENR-S04 $(O)$, (b) ENR-S08 $(\square)$ and (c) ENR-S10 $(\triangle)$. The solid curve is the best fit of Eq. (2). For clarity of plotting, the $\log E^{\prime}$ curves for ENR-S08 and ENR-S10 were displaced upward by 0.5 and 1.0 , respectively. 


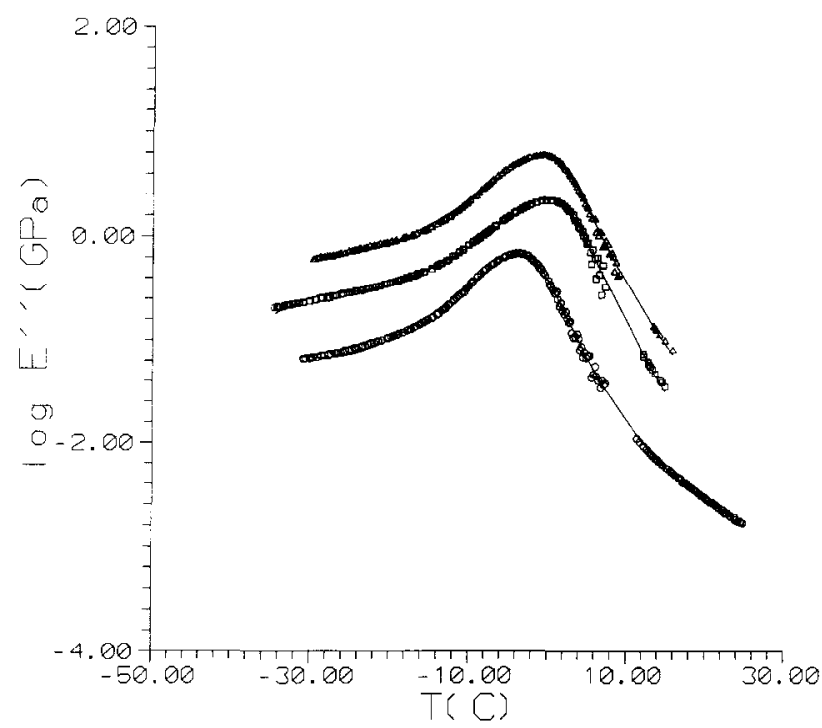

Fig. 2. Isochronal plot of the loss moduli measured for (a) ENR-S04 (O), (b) ENR-S08 $(\square)$ and (c) ENR-S10 $(\triangle)$. The solid curve is the best fit of Eq. (2). For clarity of plotting, the $\log E^{\prime \prime}$ curves for ENR-S08 and ENR-S 10 were displaced upward by 0.5 and 1.0 , respectively.

groups in the main chain. The formation of heterocyclic groups requires a neighbouring double bond. Since double bonds in the randomly epoxidized ENR-50 are isolated, the formation of intra-molecular sulfide links is inhibited $[14,16]$. Therefore, the increase in glass transition temperature with increasing sulfur content in ENR is due to the level of cross-linking. Certainly, the increase in glass transition temperature of ENR-50 is much less than that observed for NR. In Fig. 3, a normalized loss modulus $\left(E^{\prime \prime} / E^{\prime \prime}{ }_{\max }\right)$ versus $\log \left(\tau_{\max } / \tau\right)$ of samples is shown. The relaxation time $\tau$ was back-calculated from phenomenological data fitting using Eq. (2f). The subscript 'max' indicates the peak value of the loss modulus curve. As shown in Fig. 3, the normalized

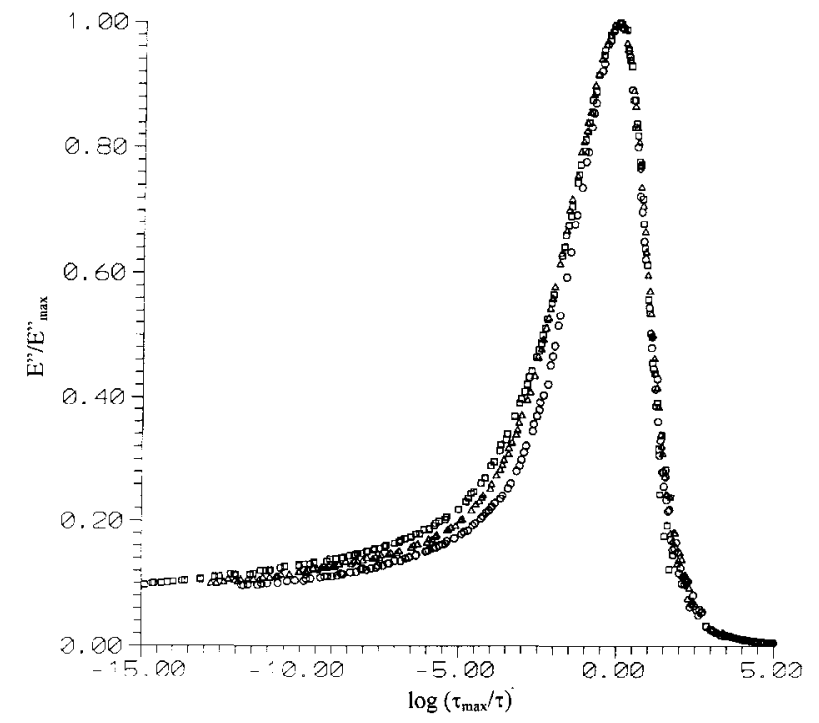

Fig. 3. Normalized loss moduli $\left(E^{\prime \prime} / E^{\prime}{ }_{\text {max }}\right)$ versus $\log \left(\tau / \tau_{\text {IIIx }}\right)$ of (a) ENRS04 (O), (b) ENR-S08 ( $\square$ ) and (c) ENR-S10 ( $\triangle$ ).

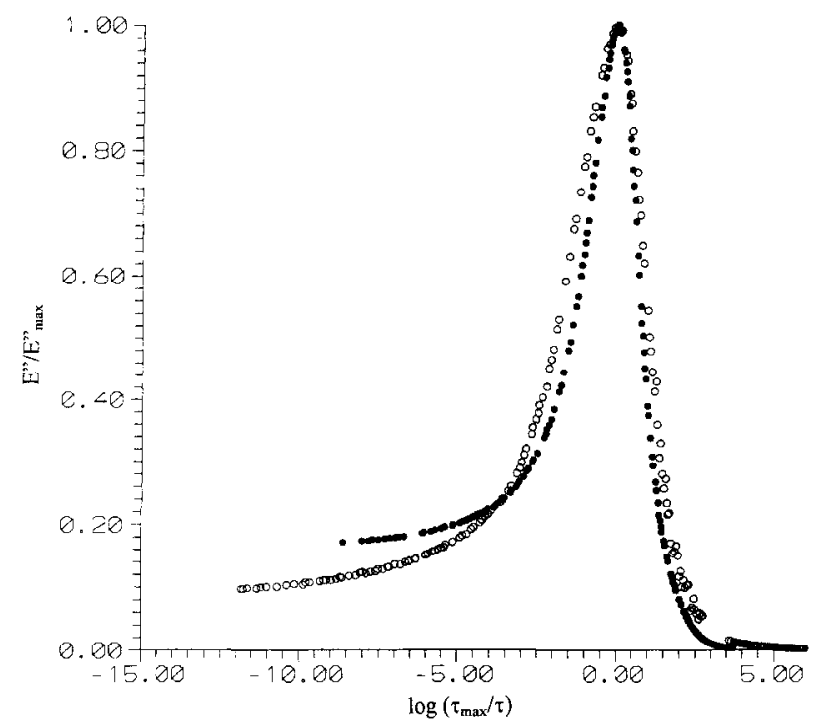

Fig. 4. Normalized loss moduli $\left(E^{\prime \prime} / E^{\prime}{ }_{\max }\right)$ versus $\log \left(\tau / \tau_{\max }\right)$ of (a) ENRS04 (O) and (b) NR04 (O).

loss modulus curves of the samples are quite similar. Since the level of cross-link is very low, the relaxation behaviour of ENR near the glass transition temperature is not changed very much by the different levels of cross-linking. Similar results were reported by Glatz-Reichenbach et al. in styrenebutyl acrylate system [27]. Fig. 4 shows a comparison of the normalized loss modulus between ENR-S04 and NR04. It is shown that NR04 has a larger low temperature (high frequency) 'tail' of the loss modulus curves. In a previous paper [10], the high frequency 'tail' of the normalized loss modulus curves decreases with increasing sulfur in the NR system. Furthermore, there is no significant difference of normalized loss modulus in the high temperature (low frequency) region.

In order to quantify the relaxation characteristics, the parameters in Eq. (2) were determined by fitting to the experimental data. The results are shown in Table 2 . As shown in Figs 1 and 2, the fitting is very well as expected.

For polymers with different chemical and physical structures, the intra- and inter-molecular interactions are different. Furthermore, the molecular motions of the polymer in the glass transition are controlled by both intra- and intermolecular interactions. The intra- and inter-molecular interactions inhibit conformational transformation and retard relaxation. Thus, the structure effect on relaxation behaviours can be examined by observing the relaxation time of molecular motions. For different polymers, the glass transition occurred in different temperature regions. A normalization scheme must be invoked to allow for comparisons among different polymers of the effect of temperature on the relaxation time. Recently, Angell [29] suggested a normalization scheme to study this effect. The relaxation time, $\tau$, is plotted as a function of temperature normalized by the glass transition temperature. Such a normalization scheme has recently been successfully utilized in polymers $[10,20]$. 
The relaxation time of the sample was back-calculated using Eq. (2f) from the relaxation spectrum fitting. Then, the relaxation time was plotted as a function of temperature normalized by the glass transition temperature measured by the dynamic relaxation experiment. The results are shown in Fig. 5. As shown in Fig. 5, the relaxation time is not changed at all with increasing concentration of sulfur in the ENR system. This result reconfirms that the relaxation behaviour is not influenced by the level of sulfur due to the low cross-linking density. In a previous paper [10], the relaxation time of NR is increased with increasing concentration of sulfur in the low temperature region and no significant difference in the high temperature region. This can be explained by the difference in structure among the samples. On a relative scale, in the high temperature region the molecular motions are expected to be large scale and inter-molecularly cooperative in nature. On the other hand, in the low temperature region the molecular motions would be expected to be small scale and very local in nature. According to Glatz-Reichenbach et al. [27], in the styrene-butyl acrylate system, the cross-link points would not result in much change of molecular motions when the concentration of the cross-link agent is less than $1 \mathrm{wt} \%$. In a previous paper [10], the concentration of sulfur in the NR system is so much less (less than $1 \mathrm{phr}$ ) that the average length of segment between cross-link points is higher than the average length of segment involved in the glass transition process [8]. It is expected that no effect of cross-linker on the molecular motions of NR will be seen. However, the formation of heterocyclic groups along the NR backbone does increase the energy barrier of intrachain molecular motions resulting in an increase of relaxation time in the low temperature region. In this study, the concentration of sulfur in the ENR system is still less than $1 \%$ phr. No cross-linking effect on the molecular motion of ENR is expected. Also, the formation of heterocyclic groups in the ENR backbone is inhibited due to the isolated double bonds as mentioned in the Introduction. Thus, it is expected that the relaxation time of ENR will not be changed at all. In Fig. 5 it is also shown that the relaxation time of ENR has a stronger temperature dependence than is observed for NR04. This is consistent with the results reported by Roland [20]. The temperature dependency of the relaxation time is related to the activation energy of molecular motions and can be calculated from $H=2.303 R A T^{2} /\left(T-T_{\infty}\right)^{2}$. In Fig. 6, the activation energy is plotted as the function of normalized temperature, $T / T_{\mathrm{g}}$. It shows that the activation energy of ENR is higher than that observed for NR04. Although no heterocyclic groups are formed in ENR during vulcanization, the epoxide groups just as the heterocyclic groups in NR increase the energy barrier of molecular motions. Furthermore, the concentration of epoxide groups in ENR is much larger than the concentration of heterocyclic groups in NR. Also, it was shown in a previous paper [10] that the activation energy for molecular motions of NR increased with increasing concentration of sulfur, i.e. the concentration 


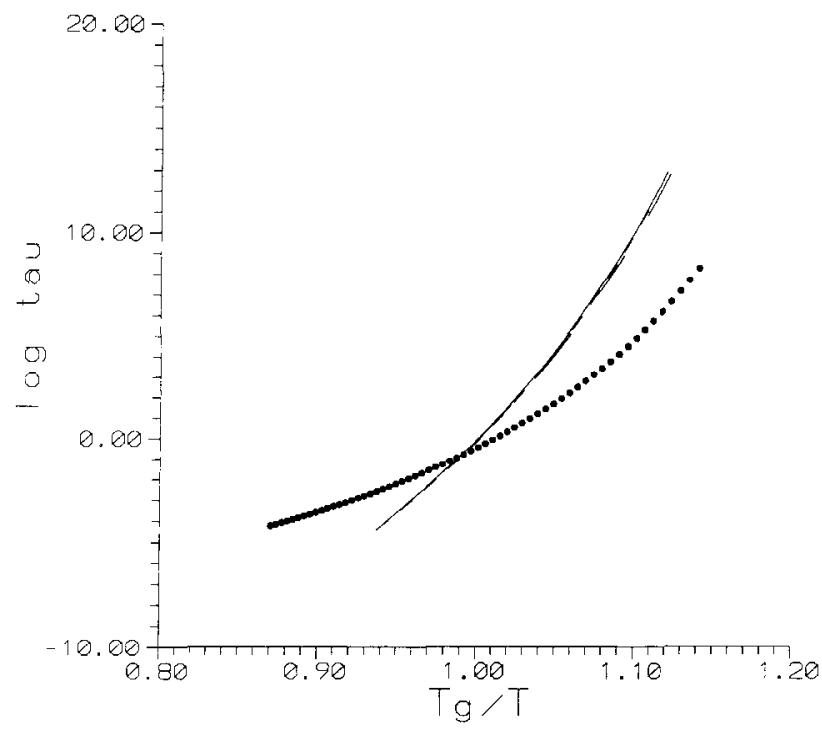

Fig. 5. The relaxation time versus $T_{\mathrm{g}}$-normalized temperature. The dashed curve with shorter segments represents the ENR-S04. The dashed curve with longer segments represents the ENR-S08. The solid curve represents the ENR-S 10. Filled symbol $(\bullet)$ represents the NR04.

of heterocyclic groups. Therefore, it is consistent that the molecular motions of ENR have higher activation energy.

The parameters, $\alpha$ and $\beta$, are back-calculated using Eq. (2c) and Eq. (2d) from the data fitting. They are not only affected by the structure of the polymer but also by the temperature. Therefore, some normalization scheme must be invoked to allow for comparisons among different polymers of the effect of temperature on $\alpha$ and $\beta$. The normalized temperature will be defined as $T-T_{\mathrm{g}}$ and the parameters are plotted versus the normalized temperature. In Fig. 7, the values of $\alpha \beta$ are plotted as a function of $T-$ $T_{\mathrm{g}}$. According to the dynamic model proposed by Schönhals and Schlosser [24-26], in the high frequency (low temperature) region, the loss dielectric constants, $\varepsilon^{\prime \prime}(\omega)$ can be scaled as

$\varepsilon^{\prime \prime}(\omega) \propto(\omega \tau)^{-n}$

where $n=\alpha \beta$ in this study. In the high frequency (low temperature) region, the molecular motions would be expected to be intra-chain and very local in nature. It is not unexpected that the values of the product $\alpha \beta$ are about the same for ENR-S04, ENR-S08 and ENR-S10 (Fig. 7). Obviously, the level of cross-linking is so low that there is no effect on the local molecular motions. This is the same as the result reported by Glatz-Reichenbach et al. [27]. GlatzReichenbach reported that in the styrene-butyl acrylate system, the values of the product $\alpha \beta$ were not changed at all in the low level of cross-linking. As shown in Fig. 7, the values of the product $\alpha \beta$ in the ENR system are less than those observed for NR04. It was suggested that in the low temperature region, the distribution of the relaxation time of ENR might exhibit broader dispersions due to the heterogeneous chain structure. Although the formation of

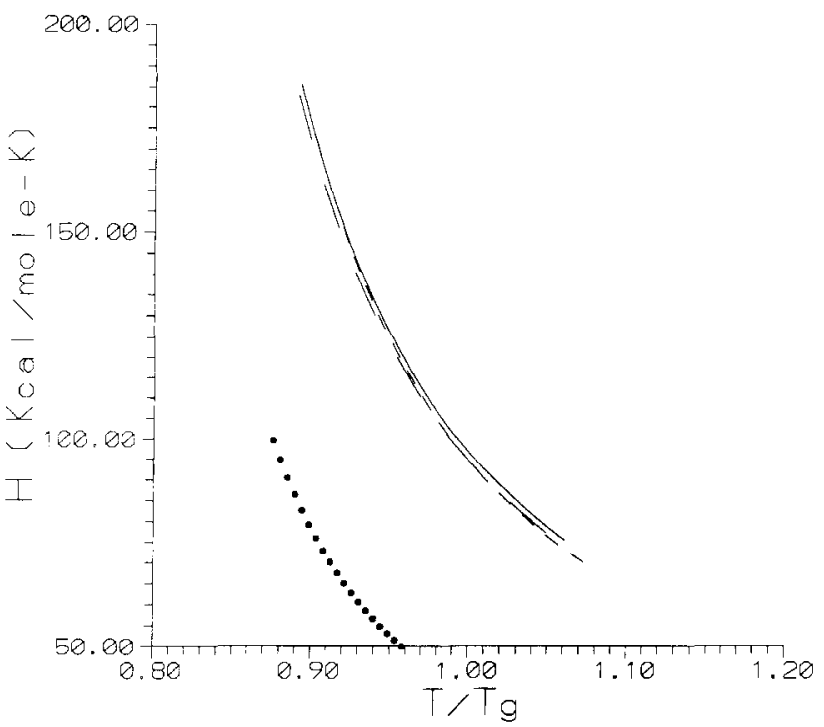

Fig. 6. The activation energy of relaxation versus $T_{\mathrm{g}}$-normalized temperature. The dashed curve with shorter segments represents the ENR-S04. The dashed curve with longer segments represents the ENR-S08. The solid curve represents the ENR-S10. Filled symbol (๑) represents the NR04.

heterocyclic groups in NR results in broader dispersions of relaxation time [10], the effect is not as strong as that by epoxide groups due to a much lower concentration of heterocyclic groups. In Fig. 8, the values of $\alpha$ were plotted as a function of $T-T_{\mathrm{g}}$. According to the dynamic model proposed by Schönhals and Schlosser [18], in the low frequency (high temperature) region the loss dielectric constants, $\varepsilon^{\prime \prime}(\omega)$, can be scaled as

$\varepsilon^{\prime \prime}(\omega) \propto(\omega \tau)^{m}$

where $m=\alpha$ in this study. In the low frequency (high temperature) region the molecular motions are expected to

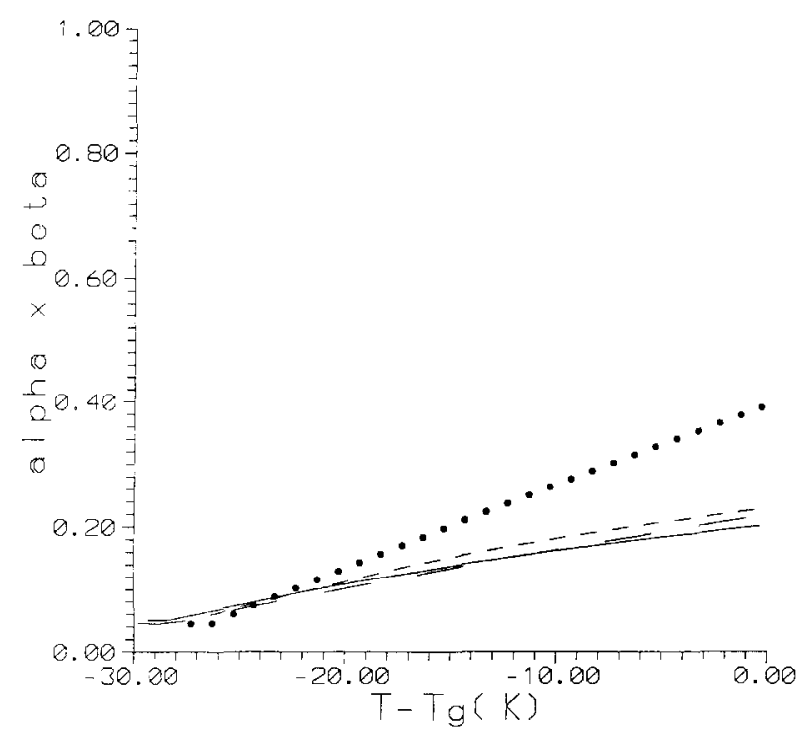

Fig. 7. The fitting parameters $\alpha \beta$ versus temperature. The dashed curve with shorter segments represents the ENR-S04. The dashed curve with longer segments represents the ENR-S08. The solid curve represents the ENRS10. Filled symbol $(O)$ represents the NR04. 


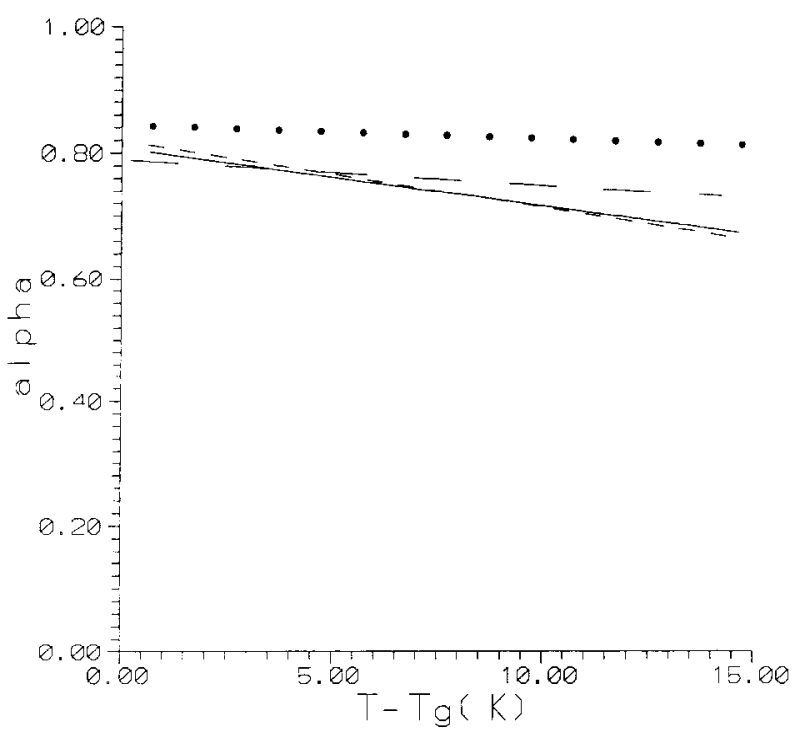

Fig. 8. The fitting parameters $\alpha$ versus temperature. The dashed curve with shorter segments represents the ENR-S04. The dashed curve with longer segments represents the ENR-S08. The solid curve represents the ENRS10. Filled symbol (-) represents the NR04.

be inter-chain and large-scale motions in nature. Again, in the high temperature region, the difference of $\alpha$ for ENR samples is not significant (the magnitude of $\alpha$ is not the same as $\alpha \beta$ ). This is consistent with the idea that the molecular motions in both the low and high temperature regions are not affected by the low level of sulfur concentration in the ENR system. The result is the same as the styrene-butyl acrylate system reported by GlatzReichenbach. The level of sulfur is too low to have a significant effect on the large scale motions. However, the values of $\alpha$ in the ENR system are less than those observed for NR04. Again, this suggests that the epoxide groups of ENR will amplify the degree of inter-molecular cooperation of motions and broaden the dispersion of relaxation time.

\section{Conclusions}

We have investigated the sulfur-concentration effect on the molecular dynamics of ENR polymers. Also, the relaxation behaviours of ENR and NR are compared to understand the structure effect on molecular motions.

The results show that the $\mathrm{HN}$ equation could describe the relaxation spectra very well. Contrary to the NR system, the relaxation time, activation energy, $\alpha$, and $\alpha \beta$ of ENR are not significantly influenced by an increasing concentration of sulfur. This suggests that such a low level of cross-linking will not affect the molecular motions in either the low or high frequency regions. The reason for the different sulfur effect on the relaxation behaviours of ENR and NR is that there is no heterocyclic group formed in ENR during the vulcanization.
On the basis of the model proposed by Schönhals and Schlosser, the epoxide groups will influence the relaxation of both the low and high frequency regions. Due to the steric interactions promoted by the epoxide groups and the enhancement of inter-molecular cooperative motions, ENR has a larger activation energy of molecular motions. From the analysis of parameters $\alpha$ and $\alpha \beta$, it is shown that epoxide groups will broaden the distribution of the relaxation time not only for the low frequency but also for the high frequency region.

\section{Acknowledgements}

The author is indebted to the Chung Shan Institute of Science and Technology for financial support of this work through the grant number: CS 85-0210-D-002-002. Thanks are also due to Dr. K. C. Cheng, Mr. W. G. Hwang and Mr. S. S. Wang for sample preparation.

\section{References}

[1] Saffer A, Johnson BL. Ind Eng Chem 1948;40:538.

[2] Gelling IR, Smith F. In: Proc Int Rubb Conf, Venice. Rubber Research Institute of Malaysia, Kuala Lumpar, 1979:140.

[3] Baker CSL, Gelling IR, Newell R. Rubb Chem Technol 1985;58:67.

[4] Davies CKL, Gelling IR, Thomas AG, Wolfe SV. Polymer 1983;24:107.

[5] Farmer EH, Shipley FW. J Chem Soc, Pt2 1947:1519.

[6] Bloomfield GF. J Chem Soc 1947:1547.

[7] McCrum NG, Read BE, Williams G. Anelastic and dielectric effects in polymeric solids. New York: Dover, 1991.

[8] Hedvig P. Dielectric spectroscopy of polymers. Bristol: Adam Hilger, 1977.

[9] Zaper AM, Koenig JL. Rubber Chem Technol 1987;60:252.

[10] Liau WB, Cheng KC. Polymer 1998, in press.

[11] Schmieder K, Wolf K. Kolloid Z 1953;134:149.

[12] Scott AH, McPherson AT, Curtis HL. J Nat Bur Stds Washington 1933;11:373.

[13] Schallamach A. Trans Inst Rubber Ind 1951;27:40.

[14] Gelling IR, Morrison NJ. Rubber Chem Technol 1985;58:243.

[15] Harris RK, Say BJ, Ng S. Polymer International 1996;40:63.

[16] Akiba M, Hashim AS. Prog Polym Sci 1997;22:475.

[17] Havriliak S, Negami S. J Polym Sci Part C 1966;14:99.

[18] Williams G, Watts DC. Trans Faraday Soc 1970;66:80.

[19] Williams G, Watts DC, Dev SB, North AM. Trans Faraday Soc 1971;67:1323.

[20] Roland CM. Macromolecules 1992;25:7031.

[21] Debye P. Polar molecules. New York: Chemical Catalogue Co., 1929.

[22] Boyd RH. Polymer 1985;26:323. 1123.

[23] Boyd RH. Macromolecules 1984;17:903.

[24] Schönhals A, Schlosser E. Collect Polym Sci 1989;267:125.

[25] Schönhals A, Schlosser E. Collect Polym Sci 1989;267:133.

[26] Schönhals A, Schlosser E. Collect Polym Sci 1989;267:963.

[27] Glatz-Reichenbach JKW. Sorriero LJ, Fitzgerald JJ. Macromolecules $1994 ; 27: 1338$.

[28] Brydson JA. Rubbery materials and their compounds. London: Elsevier Science, 1988, Chap. 4.

[29] Angell CA. J Non-Cryst Solids 1991;13:131-133. 